\title{
Effective Treatment of Paradoxical Insomnia with Acupuncture: A Case Report
}

\author{
CongFu ${ }^{1}, H_{u a n Y u}{ }^{2}$, and Yun-feiChen ${ }^{1 *}$ \\ ${ }^{1}$ Laboratory Center of Medicine, Shanghai University of Traditional Chinese Medicine, China \\ ${ }^{2}$ Department of Neurology, Huashan Hospital, Fudan University, China
}

Submission: August 28, 2017; Published: August 21, 2018

*Corresponding author: Huan Yu, Sleep and Wake Disorders Center and Department of Neurology, Huashan Hospital, Fudan University, No. 12 Middle Wulumuqi Road, Jingan District, Shanghai 200040, China; Email: dr.yuhuan@163.com

Yun-feiChen, Laboratory Center of Medicine, Yueyang Hospital of Integrated Traditional Chinese and Western Medicine, Shanghai University of Traditional Chinese Medicine, No.110 Ganhe Road, Hongkou District, Shanghai 200437, China; Email: icyf1968@163.com

\begin{abstract}
Objective: To report a the clinical approach and effectiveness of acupuncture in the treatment of paradoxical insomnia.

Methods: A 53-year-old woman with severe insomnia due to misperception of sleep state was observed. Acupuncture was administered in the patient's treatment plan.

Results: The symptom was completely controlled by acupuncture after ten-session treatment and maintained at a 6 months follow-up visit, in spite of being unresponsive to hypnotic and antidepressant pharmacotherapy previously.

Conclusion: Acupuncture may provide a new option for paradoxical insomnia.

Keywords: Paradoxical Insomnia; Acupuncture; Polysomnography; Clinical approach; Ten-session treatment; pharmacotherapy; Sleep Disorders Abbreviations: PI: Paradoxical Insomnia; CAM: Complementary and Alternative Medicine; SOL: Sleep Onset Latency; TST: Total Sleep Time; WASO: Wake Time After Sleep Onset; ICSD: International Classification of Sleep Disorders.
\end{abstract}

\section{Introduction}

Paradoxical Insomnia (PI), a less than 5\% of insomnia patients evaluated in sleep clinics [1], is a subtype of insomnia with considerable challenges related to diagnosis and treatment [2]. In International Classification of Sleep Disorders, Second Edition (ICSD-2), paradoxical insomnia is characterized by a complaint of severe sleep disturbance without corroborative objective evidence of the degree of sleep disturbance claimed [1].

Up till now, the exact pathophysiology of this misperception condition has not been identified, and efficacious treatments have not been established. Acupuncture, a component of complementary and alternative medicine (CAM), has been widely used for the treatment of insomnia in Chinese clinics, even in some western countries [3]. Since acupuncture may be effective for primary insomnia [4], we considered paradoxicalinsomnia might be responsive to it.

\section{Case}

A 53-year-old married, unemployed female presented with chronic subjective insomnia. Her presenting symptoms began 7 years ago for her annoyance at family trivialities. She first was difficult in falling asleep at night and then the symptoms aggravated. She began to take Alprazolam $0.2 \mathrm{mg}$ per night, at first her sleep condition was improved (sleep 3-4 hours after taking the medicine), however it has become worse after several days. She complained of "none of the sleep for at least five days" frequently in three months, unresponsive to alprazolam $0.4 \mathrm{mgor}$ quetiapine $25 \mathrm{mg}$ at bedtime. She reported her sleep onset latency (SOL) was more than 2-3 hours and the total sleep time (TST) was less than 2 hours per night, despite spending 8-9 hours in bed. She claimed she was tired all day but could do the housework. Her psychiatric history and mental status examination were without suggestion of psychiatric illness. Moreover, the score on Beck Anxiety Inventory and Beck depression rating scale was 27 and 13 respectively, which were all well within normal limits.

We then arranged a over-night PSG test to investigate her real sleep condition after she stopped taking any medicine for two weeks. Although she reported she had not slept on the night of the test, the objective TST derived from PSG was 445.5 minutes, SOL was 6.5 minutes, and her wake time after sleep onset (WASO) was just 28 minutes. Her sleep efficiency was 
92.8\% with normal sleep architecture without signs of apnea or periodic limb movements. On the whole, the patient met the ICSD-2 criteria for PI. She was informed about the PSG results and we recommended acupuncture treatment for her condition and she accepted.

The diagnosis of traditional medicine is shown below. Tongue: pale with teeth marks and thin white coating. Pulse: a wiry thready pulse. Syndrome differentiation: liver depression and blood deficiency. Discriminate analysis: The function of the liver isto regulate the spirit and ensure the smooth flow of Qi. The liver qi stagnation may lead to the deficiency of liver blood which would develop to the deficiency of heart blood. It then may cause the homeless of mind and underlying the insomnia.

\section{Treatment}

Participants in acupuncture group were in supine position for needling at Baihui (GV20),Yintang (GV29), bilateral Shenmen (HT7), Zusanli (ST36), Hegu (LI4), Taichong (LR 3), Zhaohai (KI 6) and Taixi (KI 3). The needles were retained for 20 minutes before removed. She was treated 3 times per week and after10 times with the same standard treatment, her symptoms of insomnia subsequently improved for describing 7 hours nocturnal sleep. This improvement persisted for the next 6 months and she was not on any medication. No tolerance phenomenon or adverse events were evident after each session and six-month follow-up.

\section{Discussion}

To our knowledge, this appears to be the first published case of paradoxical insomnia responsive to acupuncture treatment. In clinical practice, we found most PI patients did not respond to typical insomnia treatments such as non-benzodiazepine and benzodiazepine agents. The published paper showed electro convulsive therapy [5], olanzapine [2], sleep education [6] might be viable treatment options for paradoxical insomnia. Overall, efficacious treatments have not been established. In this case, her insomnia complaint had been refractory to pharmacotherapy before acupuncture, including alprazolam and quetiapine, and this mis-perception of sleep normalized with the acupuncture treatment and maintained at a 6 months follow-up visit. An improvement in the subjective complaints of sleep was noted, which suggests that acupuncture may be considered as an effective insomnia treatment, specifically for misperception of sleep state. If replicated in a well-designed cohort trial, acupuncture for PI may represent a new therapeutic option for this complicated disorder.

\section{References}

1. Medicine AAoS (2005) International Classification of Sleep Disorders. Westchester, IL: American Academy of Sleep Medicine. 2nd ed.

2. Khazaie H, Rezaie L, Darvishi F, Najafi F, Avis K (2013) Treatment of paradoxical insomnia with atypical antipsychotic drugs. A comparison of olanzapine and risperidone. Neurosciences 18(1): 64-69.

3. Burke A, Upchurch DM, Dye C, Chyu L (2006) Acupuncture use in the United States: findings from the National Health Interview Survey. Journal of alternative and complementary medicine 12(7): 639-648.

4. Cheuk DK, Yeung WF, Chung KF, Wong V (2012) Acupuncture for insomnia. The Cochrane database of systematic reviews 9: CD005472.

5. Case K, Hurwitz TD, Kim SW, Cramer-Bornemann M, Schenck CH (2008) A case of extreme paradoxical insomnia responding selectively to electroconvulsive therapy. Journal of clinical sleep medicine: JCSM: official publication of the American Academy of Sleep Medicine 4(1): 62-63.

6. Geyer JD, Lichstein KL, Ruiter ME, Ward LC, Carney PR, et al. (2011) Sleep education for paradoxical insomnia. Behavioral sleep medicine 9(4): 266-272.

\section{Your next submission with Juniper Publishers will reach you the below assets}

